\title{
Antagonism and synergism in Gardnerella vaginalis strains isolated from women with bacterial vaginosis
}

\author{
G. S. Teixeira, ${ }^{1}$ K. L. K. Soares-Brandão, ${ }^{1}$ K. M. G. R. Branco, ${ }^{1}$ \\ J. L. M. Sampaio, ${ }^{2}$ R. M. D. Nardi, ${ }^{1}$ M. Mendonça, ${ }^{3}$ R. B. Almeida, ${ }^{4}$ \\ L. M. Farias, ${ }^{1} \dagger$ M. A. R. Carvalho ${ }^{1} \dagger$ and J. R. Nicoli ${ }^{1} \dagger$ \\ ${ }^{1}$ Departamento de Microbiologia, Instituto de Ciências Biológicas, Universidade Federal de Minas \\ Gerais, Belo Horizonte, MG, Brazil \\ ${ }^{2}$ Laboratório Fleury, São Paulo, SP, Brazil \\ ${ }^{3}$ Departamento de Ginecologia e Obstetrícia, Faculdade de Medicina, Universidade Federal de \\ Minas Gerais, Belo Horizonte, MG, Brazil \\ ${ }^{4}$ Departamento de Propedêutica Complementar, Faculdade de Medicina, Universidade Federal de \\ Minas Gerais, Belo Horizonte, MG, Brazil
}

Correspondence

J. R. Nicoli

jnicoli@icb.ufmg.br

Received 17 February 2010

Accepted 11 May 2010

\begin{abstract}
Antagonistic and synergistic substances are important for interactions between micro-organisms associated with human body surfaces, either in healthy or in diseased conditions. In the present study, such compounds produced by Gardnerella vaginalis strains isolated from women with bacterial vaginosis (BV) were detected in vitro and the antagonistic ones were partially characterized. Among $11 \mathrm{G}$. vaginalis strains tested, all showed antagonistic activity against at least one of the 22 indicator bacteria assayed. Interestingly, for some of these strains, antagonism reverted to synergism, favouring one of the indicator strains (Peptostreptococcus anaerobius) when the growth medium was changed. Partial characterization of antagonistic substances suggested a bacteriocin-like chemical nature. Depending on growth conditions, G. vaginalis isolated from women with BV produced antagonistic or synergistic compounds for other bacterial components of the vaginal ecosystem. This is the first report to our knowledge of the production of antagonistic and/or synergistic substances by $G$. vaginalis. This ability may be a pivotal factor in understanding BV and the ecological role of this bacterium in the vaginal environment.
\end{abstract}

\section{INTRODUCTION}

Bacterial vaginosis (BV) is a polymicrobial syndrome where indigenous Lactobacillus populations, which are usually dominant in the vagina of healthy women (Hillier, 2005), are replaced by a mixture of bacteria which generally includes Gardnerella vaginalis, Prevotella/Bacteroides species, Peptostreptococcus species, Mycoplama hominis, Ureaplasma urealyticum and Mobiluncus species (Atassi et al., 2006). All of these bacteria can be present in low populations in healthy women, but during BV their concentrations are generally increased 100-1000-fold over normal levels (Spiegel et al., 1980). Which micro-organisms within the mixture associated with BV are essential for the syndrome or whether a single member may be the aetiological agent has never been resolved (Forsum et al., 2005). Factors which lead to the decline of the lactobacilli and the overgrowth of an atypical

†These authors contributed equally to this work.

Abbreviation: BV, bacterial vaginosis. microbiota, as well as the sequence of bacterial population changes leading to BV, also remain unknown. Microbial interactions can probably modify the environment so that it becomes adverse for some micro-organisms by production of inhibitory compounds and suitable for other organisms by releasing specific growth factors (Pybus \& Onderdonk, 1999).

Bacterial interactions, including synergism, commensalism and antagonism, are likely to play an important role in the ecology of the microbiota found on the human body surface. These phenomena may govern the atypical microbial population shifts observed during the development of BV. Pybus \& Onderdonk (1999) described in vitro nutritional inter-relationships that can explain commensalism between Prevotella bivia and both G. vaginalis and Peptostreptococcus anaerobius. However, production of antagonistic substances may also be an important factor in the competitive colonization of the vaginal ecosystem. The lactobacilli metabolize glucose 
essentially to lactic acid, which contributes to the maintenance of a low vaginal $\mathrm{pH}(4.0-4.5)$ and reduces the growth of most pathogenic micro-organisms (Aroutcheva et al., 2001). Among other antagonistic mechanisms, bacteriocinogenic activity is one of the most studied, and seems to contribute to the intra- and interregulation of the human microbiota, influencing microbial invasion and defence (Riley \& Wertz, 2002). It has also been demonstrated that methodological factors such as medium composition, culture conditions (incubation atmosphere, $\mathrm{pH}$, temperature) and bacterium growth phase may affect the expression of bacteriocin production (Lima et al., 2002).

G. vaginalis, the only species recognized in the genus Gardnerella, is a fastidious, $\beta$-haemolytic, catalase-negative, non-motile, non-capsulated, rod-shaped bacterium. This Gram-negative/variably Gram-staining facultative anaerobic bacterium depends on a $5 \% \mathrm{CO}_{2}$ atmosphere for in vitro growth (Jarosik et al., 1998) and is commonly found in the vaginal mucosa of asymptomatic women, but shows high concentrations in BV (Ingianni et al., 1997). There are no reports in the literature on production of synergistic or antagonistic compounds by $G$. vaginalis.

The present study focused on evaluating the in vitro production of antagonistic and synergistic compounds by G. vaginalis strains isolated from women with BV.

\section{METHODS}

Bacterial strains. Ten isolates of G. vaginalis isolated from women with BV and a reference strain (ATCC 14018) were tested for their inhibitory and/or stimulatory activity. The clinical isolates were identified according to Piot et al. (1982). The isolates were tested against themselves and against 22 indicator strains. Two groups of indicator strains were used: six strains pertaining to a eubiotic vaginal ecosystem (Lactobacillus); and 16 strains generally associated with a dysbiotic vaginal ecosystem (see Table 1).

In vitro antagonistic or synergistic assay. Antagonism or synergism phenomena were detected by the double layer diffusion method (Booth et al., 1977). To evaluate the influence of culture medium composition on these activities, G. vaginalis strains were grown in Brucella broth (BB) supplemented with $10 \%$ horse-blood serum, $3 \%$ meat extract and $1 \%$ proteose peptone for $48 \mathrm{~h}$, at $37{ }^{\circ} \mathrm{C}$, in an anaerobic chamber (Forma Scientific) containing an atmosphere of $85 \% \mathrm{~N}_{2}, 10 \% \mathrm{H}_{2}$ and $5 \% \mathrm{CO}_{2}$. Then the cultures were spotted with a Steers replicator onto the surface of the different culture media (see Fig. 1) using as base Brucella agar (BA), brain heart infusion agar (BHIA), Columbia agar (CA) and tryptic soy agar (TSA). After incubation at $37{ }^{\circ} \mathrm{C}$ for $48 \mathrm{~h}$ under anaerobic conditions, the strains were killed by exposure to chloroform for $30 \mathrm{~min}$. Residual chloroform was allowed to evaporate and the test cultures were overlaid with $4 \mathrm{ml} \mathrm{BB}$ soft agar $(0.75 \%)$ supplemented with $10 \%$ horse-blood serum, which had been inoculated with $0.2 \mathrm{ml}$ of a $48 \mathrm{~h} \mathrm{BB}$ culture of the indicator strain. Plates were incubated for an additional $24-48 \mathrm{~h}$ period at $37^{\circ} \mathrm{C}$ under an appropriate atmosphere for the indicator strain, and then evaluated for the presence of growth inhibition or stimulation zone. Autoantagonism was defined as the inhibition of a $G$. vaginalis strain against itself; isoantagonism as inhibition of a $G$. vaginalis strain against another $G$.
Table 1. In vitro frequency of auto-, iso- and heteroantagonistic activity of 11 Gardnerella vaginalis strains against potentially pathogenic or Lactobacillus indicator strains

Antagonistic assays were performed on BHIA supplemented with $0.5 \%$ glucose under anaerobic conditions at $\mathrm{pH}$ 7.5. Reference dysbiotic indicator strains were: Mobiluncus mulieris ATCC 35239, Bifidobacterium bifidum ATCC 29521, Bifidobacterium longum ATCC 15707, Peptostreptococcus anaerobius ATCC 27337, Prevotella intermedia ATCC 25611, Bacteroides fragilis ATCC 25285, Porphyromonas gingivalis FDC 381, Enterococcus faecalis ATCC 4083, Staphylococcus aureus ATCC 29213, Escherichia coli ATCC 25922, Salmonella enterica serovar Typhimurium ATCC 14028, Pseudomonas aeruginosa ATCC 27853, Listeria monocytogenes ATCC 15303, Bacillus cereus ATCC 11778, Proteus vulgaris ATCC 6380 and Streptococcus mutans IM/ UFRJ. Reference eubiotic indicator strains were: Lactobacillus acidophilus ATCC 4356, Lactobacillus fermentum ATCC 9338, Lactobacillus brevis ATCC 367, Lactobacillus plantarum ATCC 8014, Lactobacillus delbrueckii subsp. lactis ATCC 7830 and Lactobacillus casei subsp. rhamnosus ATCC 7469. GRF, G. vaginalis ATCC strain. Results are the number of positive assays/total number of assays.

\begin{tabular}{|cccccc|}
\hline Strain & \multicolumn{5}{c|}{ Antagonism (\%) } \\
\cline { 2 - 6 } & Auto- $^{*}$ & Iso- & $\begin{array}{c}\text { Hetero- } \\
\text { (dysbiotic) }\end{array}$ & $\begin{array}{c}\text { Hetero- } \\
\text { (eubiotic) }\end{array}$ & $\begin{array}{c}\text { Mean } \\
\%\end{array}$ \\
\hline GRF & + & 80 & 75 & 50 & 68 \\
G1 & + & 30 & 75 & 50 & 52 \\
G2 & + & 70 & 56 & 17 & 48 \\
G3 & + & 40 & 69 & 17 & 42 \\
G4 & - & 30 & 13 & 33 & 25 \\
G5 & + & 80 & 56 & 0 & 45 \\
G6 & + & 40 & 69 & 0 & 36 \\
G7 & + & 70 & 19 & 50 & 46 \\
G8 & + & 40 & 50 & 17 & 36 \\
G9 & + & 50 & 69 & 50 & 56 \\
G10 & + & 30 & 69 & 33 & 44 \\
Mean & & 51 & 56 & 29 & 45 \\
\% & & & & & \\
\hline
\end{tabular}

* $+/-$, Presence/absence of growth auto-inhibition.

vaginalis strain; and heteroantagonism the inhibition of a G. vaginalis strain against a bacterial strain of another genus.

The influence of $\mathrm{pH}$ on the antagonistic or synergistic activity was determined on BHIA supplemented with $0.5 \%$ glucose (BHIA-G) at different $\mathrm{pH}$ values (from 4.5 to 7.5 with $0.5 \mathrm{pH}$ intervals). The plates were incubated in an anaerobic chamber for $48 \mathrm{~h}$ at $37^{\circ} \mathrm{C}$. The antagonistic or synergistic activity was determined as described above.

Detection of interfering factors for antagonistic activity. To exclude the eventual interference of bacteriophage action on the antagonistic phenomenon, a piece $(3 \mathrm{~mm}$ in diameter) of agar was removed aseptically from the inhibition zone, according to the method described by Turner \& Jordan (1981). The agar block was placed onto BHIA-G and recovered with $4.0 \mathrm{ml}$ BHI broth soft agar, containing the indicator strain. Plates were then incubated for $48 \mathrm{~h}$ at $37{ }^{\circ} \mathrm{C}$ under anaerobiosis, before evaluation for the presence of lytic zones. 
(a)

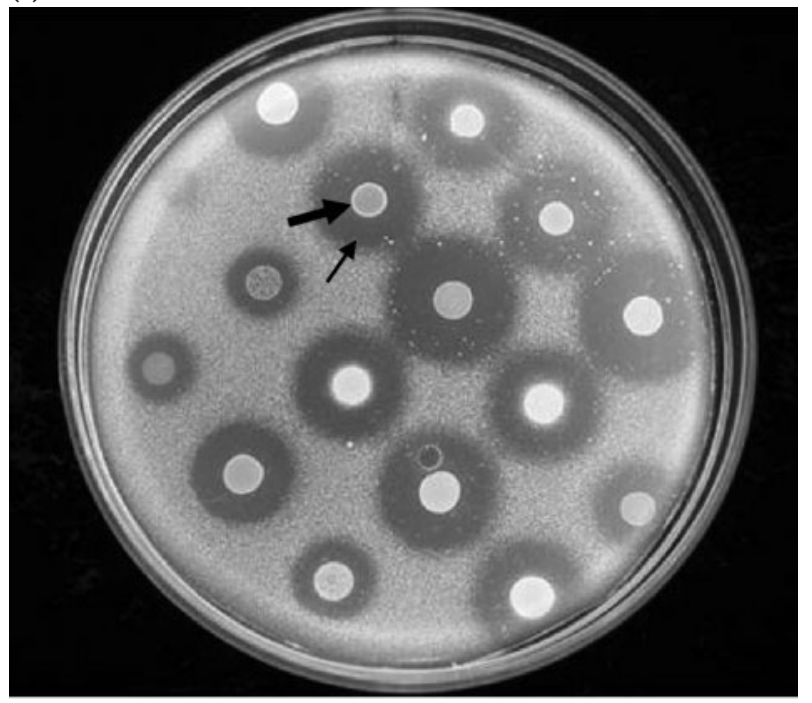

(b)

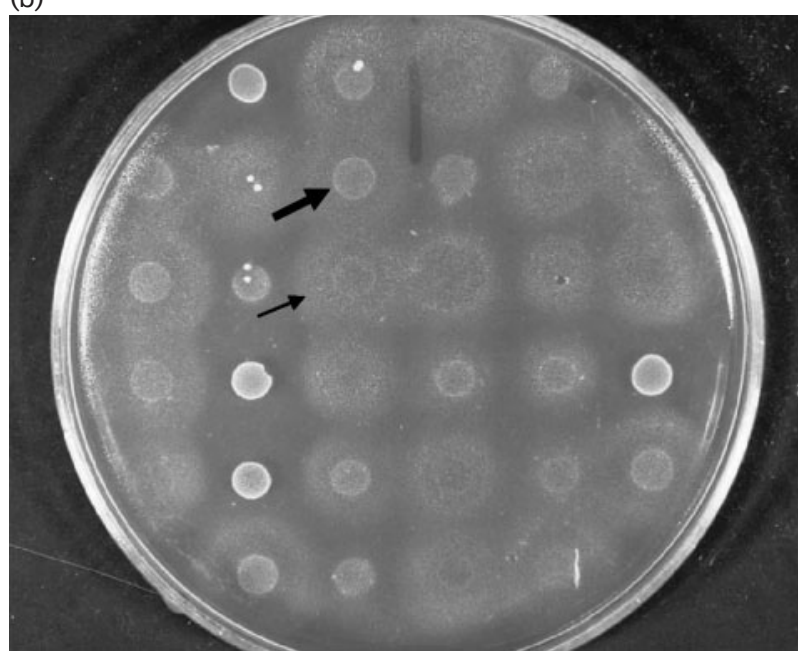

Fig. 1. Aspect of in vitro antagonism (a) and synergism (b) of $G$. vaginalis. (a) Isoantagonism between a producer strain of $G$. vaginalis and other $G$. vaginalis strains used as indicator. (b) Synergism between a $G$. vaginalis producer strain and Peptostreptococcus anaerobius ATCC 27337 as indicator. In both cases, the medium used was $\mathrm{CA}, \mathrm{pH} 7.0$, under microaerophilic and anaerobic conditions, respectively. Large arrows show the $G$. vaginalis growth spot and the smaller arrows show the inhibition (a) or overgrowth (b) halo of Peptostreptococcus anaerobius.

To search for possible inhibition by long-chain fatty acids, tests were conducted on BHIA-G with and without the addition of $1 \%$ soluble starch (Walstad et al., 1974). The pH on the inhibition zone and on the non-inoculated medium was measured using a surface microelectrode (Microelectrode Inc.).

Finally, to exclude a possible interference of residual chloroform on the growth of indicator strains, the evaluation of antagonistic activity was performed without its use. After the growth of the producer strains, the indicator strains were inoculated with a loop near the spots of the producer strains without previous exposure to chloroform. The cultures were incubated at $37{ }^{\circ} \mathrm{C}$ for $48 \mathrm{~h}$, under anaerobiosis, and then examined for the presence of inhibition zones.

Sensitivity to four proteolytic enzymes was tested as described by Farias et al. (1994). Fifty microlitres of enzyme solutions [proteinase K (Boehringer; $50 \mu \mathrm{l} \mathrm{ml}{ }^{-1}$ ); type II trypsin (Sigma; $200 \mu \mathrm{ml}^{-1}$ ); papain (Sigma; $10 \mathrm{mg} \mathrm{ml}^{-1}$ ); $\alpha$-chymotrypsin] was placed onto the agar surface around the growing spots and allowed to cover areas larger than the expected zones of inhibition. Incubation was carried out for $4 \mathrm{~h}$ at $37{ }^{\circ} \mathrm{C}$ under anaerobiosis. Two controls were run: one only with the enzyme, and the other only with the producing bacterium. Sensitivity to temperature was determined by exposure of the culture to temperatures of $37,45,60,70$ and $80{ }^{\circ} \mathrm{C}$ for $20 \mathrm{~min}$ before the assay. The effects of these conditions were determined on the basis of their activity toward the inhibitory substances produced against the indicator strain Peptostreptococcus anaerobius ATCC 27337.

Statistical analysis. All the experiments were performed in duplicate or triplicate. Data were analysed by Fisher's exact test. The level of significance was set at $P<0.05$. Statistical analyses were performed using the SigmaStat software (version 1.0; Jandel).

\section{RESULTS AND DISCUSSION}

Antagonism of Lactobacillus species from the eubiotic vaginal ecosystem against pathogenic micro-organisms responsible for BV is a well-known phenomenon (Aroutcheva et al., 2001; Atassi et al., 2006). Among these pathogenic micro-organisms, a few synergistic interactions with each other have been demonstrated (Pybus \& Onderdonk, 1999). However, there are few reports of antagonistic activity of the pathogens with each other as well as against Lactobacillus species, especially concerning G. vaginalis. The aim of the present study was to evaluate a possible production of antagonistic substances by $G$. vaginalis strains which could lead to the disappearance of lactobacilli or any other competitor and to potentially pave the way for establishment of a BV dysbiotic microbiota.

A total of 23554 assays were performed to evaluate the production of synergistic or antagonistic substances by $G$. vaginalis. Fig. 1 shows the aspect of representative assays where antagonism (Fig. 1a) and synergism (Fig. 1b) were observed. Diameters of the inhibitory zones ranged from 2 to $16 \mathrm{~mm}$, depending on the producer and indicator strains, as well as on the physico-chemical conditions of growth.

Table 1 shows that autoantagonism was noted for all the strains except G4. When used as indicator, this strain did not show sensitivity to isoantagonism, and when used as producer showed one of the lower antagonistic activities (30\%), together with G1 and G10. Some of the strains, such as G6 (100\%), G1 (91\%) and G10 (91\%), were highly sensitive to isoantagonism. Concerning isoantagonism, reference and G5 strains showed the higher activity $(80 \%)$. All the G. vaginalis strains produced heteroantagonistic substances against at least one of the dysbiotic indicator strains. This heteroantagonistic activity was higher for the reference (GRF) and G1 strains (75\%), 
and again lower for the G4 strain. Among the 16 dysbiotic indicator strains, Peptostreptococcus anaerobius was the most sensitive as demonstrated by the frequency of inhibition (100\%) and the mean diameter of the inhibition zone $(11.5 \mathrm{~mm})$. High sensitivity was also observed for Proteus vulgaris (91\%), Staphylococcus aureus (82\%), Bacillus cereus ( $82 \%)$ and Bacteroides fragilis (82\%). However, Enterococcus faecalis and Streptococcus mutans did not exhibit sensitivity under any of the conditions studied. Table 1 also shows antagonistic activity of $G$. vaginalis against eubiotic indicator strains (Lactobacillus). Lactobacillus acidophilus was the most sensitive indicator strain, whereas Lactobacillus brevis and Lactobacillus plantarum were totally resistant to the antagonistic activity of the G. vaginalis strains. Among these last strains, the highest frequencies (50\%) of antagonistic activity were produced by strains G1, G7, G9 and the reference strain (GRF). Two G. vaginalis strains (G5 and G6) did not show antagonistic activity against the Lactobacillus strains tested. Globally, the GRF strain showed the highest antagonistic activity (68\%) and G4 strain the lowest (25\%). Iso- (51\%) and heteroantagonism against dysbiotic indicator strains $(56 \%)$ were more frequent than heteroantagonism against eubiotic indicator strains (29\%).

Most women with BV are frequently and heavily colonized by $G$. vaginalis, but the exact role of this bacterium in the development of the syndrome is not well understood. It is accepted that the levels of $G$. vaginalis in the vaginal site may be controlled by antagonism or synergism interactions between the different microbial species present, but the production of inhibitory compounds has never been described for this bacterium. The present study demonstrated that various $G$. vaginalis strains produced an inhibitory diffusible substance(s) with auto-, iso- and heteroantagonism against a wide range of bacteria. The selection of the indicator strains was based on their importance in the vaginal ecosystem. Additionally, Salmonella Typhimurium, Pseudomonas aeruginosa, Listeria monocytogenes, Bacillus cereus, Proteus vulgaris, Streptococcus mutans, Enterococcus faecalis, Staphylococcus aureus and Escherichia coli were also included in the study to evaluate the spectrum of action of the antagonistic substance, and because some of them could eventually be transient organisms of the vagina. Apparently, antagonistic capacity was not indispensable for the development of BV, since among the $11 \mathrm{G}$. vaginalis strains isolated from vaginal ecosystem of women affected by the syndrome, some of them showed a high antagonistic activity (56\% for G9) whereas others presented a low capability (25\% for G4). Interestingly, the higher activity of G1 was associated with a high sensitivity to isoantagonism (91\%) whereas G4 was simultaneously less sensitive and less productive. Concerning the indicator strains, the higher sensitivity of Peptostreptococcus anaerobius to the antagonistic activity of G. vaginalis was also observed when oral Fusobacterium species (Oliveira et al., 1998), Actinobacillus actinomycetemcomitans (Lima et al., 2002) and Eikenella corrodens (Apolônio et al., 2007) were used as producer strains. The antagonism observed against the Lactobacillus species could explain the substitution of this predominant bacterial genus regularly found in the eubiotic vaginal ecosystem by the dysbiotic microbiota found in BV. However, as pondered above, this heteroantagonistic activity was not found for all the $G$. vaginalis strains tested.

Fig. 2 shows the influence of culture medium on the mean frequency of antagonism for the 11 G. vaginalis strains, using Peptostreptococcus anaerobius as the indicator strain. Antagonist activity was best detected when BHIA and CA

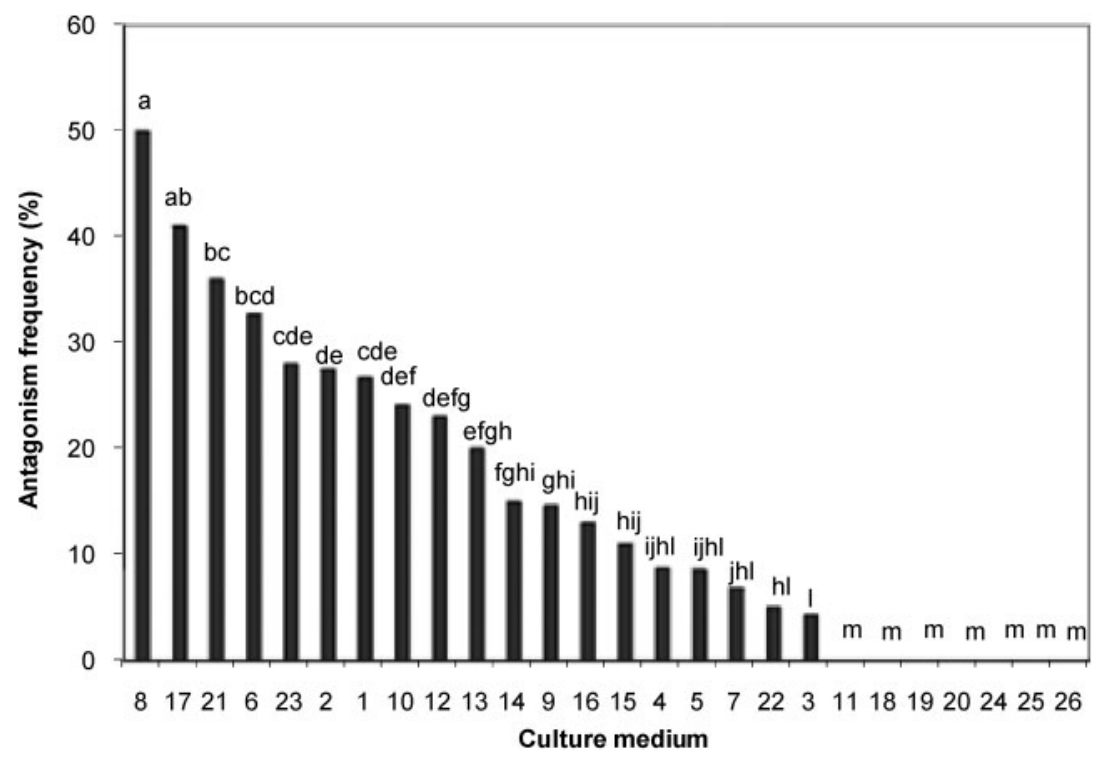

Fig. 2. Influence of culture media on the antagonistic activity frequency of $11 \mathrm{G}$. vaginalis strains. The following media were used: 1 , BHIA with $0.1 \%$ glucose; $2, \mathrm{BHIA}$ with $0.5 \%$ yeast extract; 3 , BHIA with $0.4 \%$ sodium bicarbonate and $1 \%$ yeast extract; 4 , $\mathrm{BHIA}$ with $1 \%$ glucose; 5 , BHIA with $0.4 \%$ sodium bicarbonate; 6 , BHIA; 7, BHIA with 0.1 yeast extract; 8 , BHIA with $0.5 \%$ glucose; 9 , BHIA with $1 \%$ yeast extract; 10, CA with $1 \%$ proteose peptone; $11, \mathrm{CA} ; 12, \mathrm{CA}$ with $1 \%$ yeast extract; $13, \mathrm{CA}$ with $0.4 \%$ sodium bicarbonate; 14, BA; 15, BA with $1 \%$ yeast extract; $16, \mathrm{BA}$ with $1 \%$ glucose; $17, \mathrm{CA}$ with $0.1 \%$ glucose; 18 , CA with $0.5 \%$ glucose; 19 , BA with $0.5 \%$ glucose; 20, BA with $0.4 \%$ sodium bicarbonate; 21, BHIA with $1 \%$ proteose peptone; 22 , BA with $1 \%$ proteose peptone; 23, CA with $1 \%$ glucose; $24, \mathrm{BA}$ with $0.1 \%$ glucose; 25 , TSA; 26, TSA with $0.5 \%$ glucose. Different letters $(a-m)$ indicate significant difference $(P<0.05)$. 
were used. Supplementation with glucose, yeast extract and/or sodium bicarbonate did not show any consistent pattern of enhancement of the antagonistic activity. The best and similar $(P>0.05)$ results were observed for BHIA and CA supplemented with $0.5 \%$ and $0.1 \%$ glucose, respectively. Antagonism was not observed when TSA was used, and a low frequency of this phenomenon was noted with BA.

During the experiments carried out to determine the influence of growth medium using Peptostreptococcus anaerobius as indicator strain, a reversion of antagonistic to synergistic inter-relationships was observed depending on the culture medium. Table 2 shows this synergistic activity of $G$. vaginalis strains enhancing the growth of Peptostreptococcus anaerobius on TSA medium. This phenomenon was enhanced when TSA was supplemented with $0.5 \%$ glucose, except at a lower (5.0) and higher (7.5) $\mathrm{pH}$.

It is well known that the production of antagonistic substance may be influenced by culture conditions such as medium composition, incubation atmosphere, $\mathrm{pH}$, temperature and the growth phase of the micro-organism (Lima et al., 2002). In the present study, among different combinations of media and supplements tested, higher production of antagonistic substance was observed when nutritionally rich culture media were used (BHIA and CA) when compared to media presenting lower nutritional value (TSA).

Another interesting and unexpected fact observed during the present study on growth conditions was their influence on the types of inter-relationships between the producer and the indicator strains. As described above, antagonism of $G$. vaginalis against Peptostreptococcus anaerobius observed on BHIA and CA media was not found when TSA was used as growth medium. Surprisingly, in this last situation, a stimulation of Peptostreptococcus anaerobius growth was obtained around the G. vaginalis spot. However, these results are in agreement with data from the literature showing that production of inhibitory compounds, such as bacteriocin-like substances, needs nutritionally rich culture medium. Additionally, on a nutritionally poor culture medium, such as TSA (when compared to BHIA and CA), growth of more fastidious bacteria could be enhanced by growth factors produced by another bacterium. This might be the basis of the polymicrobial aetiology observed during BV with a clear association between $G$. vaginalis and various anaerobes (Pybus \& Onderdonk, 1999; Cauci et al., 2003; Eschenbach, 2007). Indeed, it is known that G. vaginalis produces amino and keto acids, such as succinate, which are considered growth factors for anaerobic bacteria.

The assay to detect the presence of bacteriophage in the inhibition zone was negative, indicating that antagonism was not due to the presence of a phage. The possibility of inhibition by long-chain fatty acids was also rejected because of the lack of the interference upon addition of soluble starch in the medium. No significant variations in $\mathrm{pH}$ values were detected inside and outside the inhibition zones, or in the non-inoculated medium. The experiments carried out without the use of chloroform gave the same results as those that included exposure to chloroform to kill the producer isolates.

Inhibitory activity was progressively reduced when exposed to temperatures of 37,45 and $60{ }^{\circ} \mathrm{C}$ for $20 \mathrm{~min}$, and was eliminated after exposure at 70 and $80{ }^{\circ} \mathrm{C}$ during the same period. The inhibitory activity produced by G. vaginalis

Table 2. In vitro synergistic activity of $11 \mathrm{G}$. vaginalis strains against Peptostreptococcus anaerobius ATCC 27337

Synergistic assays were performed on TSA supplemented or not with $0.5 \%$ glucose and incubated at $37{ }^{\circ} \mathrm{C}$ under microaerophilic conditions. GRF, G. vaginalis ATCC strain; +, presence of growth stimulation; -, absence of growth stimulation.

\begin{tabular}{|c|c|c|c|c|c|c|c|c|c|c|c|c|}
\hline \multirow[t]{2}{*}{ Strain } & \multicolumn{12}{|c|}{ Culture medium and $\mathrm{pH}$ value } \\
\hline & \multicolumn{6}{|c|}{ TSA } & \multicolumn{6}{|c|}{ TSA $+0.5 \%$ glucose } \\
\hline GRF & - & - & - & - & - & - & - & + & + & + & + & - \\
\hline G1 & + & + & + & + & + & + & - & + & + & + & + & - \\
\hline G4 & + & + & + & + & + & + & - & + & + & + & + & - \\
\hline G5 & + & + & + & + & + & + & - & + & + & + & + & - \\
\hline G6 & + & + & + & + & + & + & - & + & + & + & + & - \\
\hline G7 & + & + & + & + & + & + & - & + & + & + & + & - \\
\hline G8 & - & - & - & - & - & - & - & + & + & + & + & - \\
\hline
\end{tabular}


was lost after exposure to proteinase $\mathrm{K}$ and $\alpha$-chymotrypsin, but not when papain and trypsin were tested.

Concerning the possible chemical nature of the inhibitory substance, various studies have described the production of organic acids, hydrogen peroxide and bacteriocins by vaginal Gram-positive bacteria such as Lactobacillus species (Ocaña \& Nader-Macías, 2004; Kaewsrichan et al., 2006), Enterococcus faecium and Lactococcus lactis (Todorov et al., 2007). Based on the results of the assays performed to test the influence of growth conditions, BHIA supplemented with $0.5 \%$ glucose was selected to characterize the antagonistic activity expressed by $G$. vaginalis. The antagonism observed here for the G. vaginalis isolates was not due to chloroform action, bacteriophage, long-chain fatty acids or acidic $\mathrm{pH}$, as demonstrated by the experiments on interfering factors. The antagonistic substance or substances detected were sensitive to high temperatures and exhibited loss of activity after exposure to some of the tested proteases, which suggest a protein nature. These results also suggest a bacteriocin molecule as being responsible for the observed inhibitory effects. The new antagonist activity described in the present study for G. vaginalis also showed a wide spectrum, inhibiting both Gram-positive and Gram-negative bacteria, and for this reason seems to be bacteriocin-like. Moreover, various producer strains exhibited a different inhibition spectrum depending on the medium, $\mathrm{pH}$ and incubation atmosphere employed in the assay, which could be explained by the production of more than one antagonistic substance with different physico-chemical and biological properties.

In conclusion, the present study is the first report, to our knowledge, describing the production by $G$. vaginalis of either antagonistic or synergistic compounds depending on the growth conditions. This and further studies may be of taxonomic and epidemiological interest and might also contribute to the understanding of the role of these substances in vaginal microbial ecology. For this purpose, purification and characterization of the substances detected and others eventually produced by this bacterium will be important, and are currently under way in our laboratory.

\section{ACKNOWLEDGEMENTS}

This work was supported by grants from Coordenação de Aperfeiçoamento de Pessoal de Nível Superior (CAPES), Fundação de Amparo à Pesquisa de Minas Gerais (FAPEMIG), Conselho Nacional de Desenvolvimento Científico e Tecnológico (CNPq) and Pró Reitoria de Pesquisa da UFMG (PRPq/UFMG). The authors are indebted to Luzia R. Rezende and José Sérgio B. Souza for their technical assistance.

\section{REFERENCES}

Apolônio, A. C. M., Carvalho, M. A. R., Ribas, R. N. R., Sousa-Gaia, L. G., Santos, K. V., Lana, M. A., Nicoli, J. R. \& Farias, L. M. (2007). Production of antagonist substance by Eikenella corrodens isolated from the oral cavity of human beings with and without periodontal disease. J Appl Microbiol 103, 245-251.

Aroutcheva, A., Gariti, D., Simon, M., Shott, S., Faro, J. \& Simoes, J. A. (2001). Defense factors of vaginal lactobacilli. Am J Obstet Gynecol 185, 375-379.

Atassi, F., Brassart, D., Grob, P., Graf, F. \& Servin, A. (2006). Lactobacillus strains isolated from the vaginal microbiota of healthy women inhibit Prevotella bivia and Gardnerella vaginalis in coculture and cell culture. FEMS Immunol Med Microbiol 48, 424-432.

Booth, S. J., Johnson, J. L. \& Wilkins, T. D. (1977). Bacteriocin production by strains of Bacteroides isolated from human feces and the role of these strains in the bacterial ecology of the colon. Antimicrob Agents Chemother 11, 718-724.

Cauci, S., Thorsen, P., Schendel, E. D., Bremmelgaard, A., Quadrifoglio, F. \& Guaschino, S. (2003). Determination of immunoglobulin A against Gardnerella vaginalis hemolysin, sialidase, and prolidase activities in vaginal fluid: implications for adverse pregnancy outcomes. J Clin Microbiol 41, 435-438.

Eschenbach, D. A. (2007). Bacterial vaginosis: resistance, recurrence, and/or reinfection? Clin Infect Dis 44, 220-221.

Farias, L. M., Totola, A. H., Miranda, C. M. S., Carvalho, M. A. R., Damasceno, C. A. V., Tavares, C. A. P., Cisalpino, E. O. \& Vieira, E. C. (1994). Extraction, partial purification and characterization of a bacteriocin (fragilicin) produced by a strain of Bacteroides fragilis isolated from Callithrix penicillata. Res Microbiol 145, 9-16.

Forsum, U., Holst, E., Larsson, P. G., Vasquez, A., Jakobsson, T. \& Mattsby-Baltzer, I. (2005). Bacterial vaginosis - a microbiological and immunological enigma. APMIS 113, 81-90.

Hillier, S. L. (2005). The complexity of microbial diversity in bacterial vaginosis. $N$ Engl J Med 353, 1886-1887.

Ingianni, A., Petruzzelli, S., Morandotti, G. \& Pompei, R. (1997). Genotypic differentiation of Gardnerella vaginalis by amplified ribosomal DNA restriction analysis (ARDRA). FEMS Immunol Med Microbiol 18, 61-66.

Jarosik, G. P., Land, C. B., Duhon, P., Chandler, R., Jr \& Mercer, T. (1998). Acquisition of iron by Gardnerella vaginalis. Infect Immun 66, 5041-5047.

Kaewsrichan, J., Peeyananjarassri, K. \& Kongprasertkit, J. (2006). Selection and identification of anaerobic lactobacilli producing inhibitory compounds against vaginal pathogens. FEMS Immunol Med Microbiol 48, 75-83.

Lima, F. L., Farias, F. F., Carvalho, M. A. R., Alviano, C. S. \& Farias, L. M. (2002). Influence of abiotic factors on the bacteriocinogenic activity of Actinobacillus actinomycetemcomitans. Res Microbiol 153, 249-252.

Ocaña, V. S. \& Nader-Macias, M. E. (2004). Production of antimicrobial substances by lactic acid bacteria II: screening bacteriocin-producing strains with probiotic purposes and characterization of a Lactobacillus bacteriocin. Methods Mol Biol 268, 347-353.

Oliveira, A. A. P., Farias, L. M., Nicoli, J. R., Costa, J. E. \& Carvalho, M. A. R. (1998). Bacteriocin production by Fusobacterium isolates recovered from the oral cavity of human subjects with and without periodontal disease and of marmosets. Res Microbiol 149, 585-594.

Piot, P., Dyck, E. V., Totten, P. A. \& Holmes, K. K. (1982). Identification of Gardnerella (Haemophilus) vaginalis. J Clin Microbiol 15, 19-24.

Pybus, V. \& Onderdonk, B. A. (1999). Microbial interactions in the vaginal ecosystem, with emphasis on the pathogenesis of bacterial vaginosis. Microbes Infect 1, 285-292.

Riley, M. A. \& Wertz, J. E. (2002). Bacteriocins: evolution, ecology, and application. Annu Rev Microbiol 56, 117-137. 
Spiegel, C. A., Amsel, R., Eschenbach, D., Schoenknecht, F. \& Holmes, K. K. (1980). Anaerobic bacteria in nonspecific vaginitis. $N$ Engl J Med 303, 601-606.

Todorov, S. D., Botes, M., Danova, S. T. \& Dicks, L. M. T. (2007).

Probiotic properties of Lactococcus lactis ssp. lactis HV219, isolated from human vaginal secretions. J Appl Microbiol 103, 629-639.
Turner, J. W. \& Jordan, H. V. (1981). Bacteriocin like activity within the genus Actinomyces. J Dent Res 60, 1000-1007.

Walstad, D. L., Resistz, R. C. \& Sparling, P. F. (1974). Growth inhibition among strains of Neisseria gonorrhoeae due to production of inhibitory free fatty acids and lysophosphatidyl ethanol amine: absence of bacteriocins. Infect Immun 10, 481-488. 